\title{
Multi-parametric magnetic resonance imaging in acute necrotizing encephalopathy of children: validity and prognostic value
}

Rania S. M. Ibrahim ${ }^{1} 2^{*}$, W. Elzayat ${ }^{1}$, H. M. Seif ${ }^{1}$, H. A. El-Kiki', S. Emad-Eldin', M. Shahin' ${ }^{1}$ S. M. Kamel', R. Osama', R. Zakaryia ${ }^{1}$, M. Fatouh ${ }^{1}$ and Rania H. Hachem ${ }^{1 *}$

\begin{abstract}
Background: Acute necrotizing encephalopathy of children (ANEC) is a rare fulminant type of acute encephalopathy that mainly occurs in children with a characteristic clinico-radiological pattern. It is commonly preceded by viral infections. The condition carries a poor prognosis with high morbidity and mortality rates. We highlight the relationship between diagnostic multi-parametric magnetic resonance imaging (MRI) findings and correlate them with the clinical outcome of children with ANEC by enrollment of MRI scoring.

Results: The thalami were involved in all 30 patients, brain stem in $80 \%$, basal ganglia (13.3\%), cerebral white matter (WM) in 73\%, and cerebellar WM in 33\%. Hemorrhage was present in $86.7 \%$ patients, edema $80 \%$, and necrosis in 13.3\%. We found that the patients having the highest MRI scores were in the poor outcome category; whereas the patients with lower MR score (1 or 2 ) had better outcomes. Statistically positive correlation $(r=0.1198)$ was found between the multi-parametric MR score and the outcome category.
\end{abstract}

Conclusion: An extended multi-parametric MRI should be performed in ANEC, allowing early detection and scoring of the disease for better prognosis. There is a positive correlation between the clinical outcome and the MR scoring.

Keywords: Acute necrotizing encephalopathy, Children, Magnetic resonance imaging

\section{Background}

A rare fulminant and devastating encephalopathy (ANEC) affects infants and children with a characteristic clinicoradiological pattern are called ANEC [1]. Its etiology and pathogenesis remain unknown, although respiratory and gastrointestinal (GIT) viral infections (e.g., influenza A virus, mycoplasma, rota virus, and human herpes virus-6) have been reported as common causative agents [2]. It is recently believed that this disease is most likely immunemediated or even metabolic [3]. It has been reported that

\footnotetext{
* Correspondence: raniasaberm@hotmail.com; raniahachem@hotmail.com ${ }^{1}$ Radiology Department (Pediatric unit), Faculty of Medicine, Kasr El-Ainy Hospital, Cairo University, Kasr Al-aini Street, Al-manial District, Cairo, Egypt Full list of author information is available at the end of the article
}

cytokines, such as tumor necrosis factor receptor-1 and interleukins could mediate the disease [4].

Prodromal symptoms appear in the first stage include fever, signs of upper respiratory tract infections and gastroenteritis, and erythema [5]. With the development of ANEC in the second or mid stage, rapid and progressive symptoms of brain dysfunctions may present as convulsions, disturbance of consciousness, and focal neurological deficits [6]. Laboratory findings are variable, such as abnormalities of liver function. Moreover, the protein levels of cerebrospinal fluid (CSF) and platelet count could be a predictor of the prognosis of the disease [6]. The prognosis of ANEC is variable, from a mild form even up to a complete recovery to a severe form with a high mortality [7]. The diagnosis of ANEC was 
mainly based on characteristic neuro-radiologic findings [8] characterized by multiple, symmetrical lesions showing T2 prolongation in the thalami, frequently with accompanying lesions in the brain stem tegmentum, putamina, cerebral, and cerebellar hemispheres WM [9].

These changes resolve nearly completely on follow-up imaging within days to weeks [10]. Treatment usually includes antiviral therapy, immunomodulatory agents, and even intensive care unit (ICU) [11]. Outcomes in ANEC are poor, with mortality up to $30 \%$ of cases; various neurological impairments are frequently reported, although a good outcome with no ongoing sequelae can occur in $10 \%$ of cases [12]. The current study determines the role of multi-parametric MRI in the diagnosis and the follow-up of children with ANEC in relation to their clinical outcome with enrollment of a multi-parametric scoring system.

\section{Methods \\ Patients}

A retrospective study of 30 children with ANEC evaluated during autumn and winter seasons between October 2018 and March 2020; they were admitted to a tertiary children university hospital and were sent to the radiology department for MRI brain imaging because of seizure and confusion. The subjects consisted of 20 boys and 10 girls whose ages ranged from 8 months to 9 years (mean age, 4.32 years). The cases enrolled in this study based on the criteria of ANEC described by Mizuguchi et al. [1], which are acute encephalopathy with rapid conscious deterioration, absence of serum hyperammonemia, CSF pleocytosis, increase in CSF proteins, neuroimaging studies showing symmetrical, and multifocal lesions involving the thalami. We excluded any cases with other clinical diseases or other brain abnormalities resembling ANEC. Also, patients with any contraindication for MRI examination were excluded. The study protocol was approved by the Research Ethical Committee, University Children Hospital. All patients or patients' guardians were counseled and signed a consent form.

\section{Imaging}

The study was performed using multi-parametric MRI as follows: initial study on each of the patient and a follow-up study for survivors. The mean interval between the onset of disease and the initial study was 3.6 days, and that between the onset of disease and the follow-up study was 6 weeks. We used a MRI $1.5 \mathrm{~T}$ superconducting scanner (Magnetom Aera, Siemens Healthineers, Germany). Although various MR pulse sequences were obtained, axial T1- spin-echo-weighted images (WI) and fluid-attenuated inversion recovery (FLAIR) images, axial and coronal T2-weighted images, diffusion-weighted images (DWI) and apparent diffusion coefficient (ADC) images, DWI was performed with the $b$ values were 0,500 , and $1000 \mathrm{~s} / \mathrm{mm}^{2}$ and $\mathrm{T}_{2}$ "-weighted gradient echo imaging or susceptibility-weighted images (SWI) were available for all patients. A limited MR spectroscopy (MRS) was done for 15 cases only who were clinically fit for the long examination; we obtained single-voxel proton MRS using echo times (TE) of 115 ms. The voxel location for single-voxel MRS was set away from any hemorrhage or necrosis. We measured lactate peak-lactate complex peak intensities at $0.8-1.5$ parts per million ( $\mathrm{ppm})$, glutamate/glutamine complex peak intensities at $2.0-2.5 \mathrm{ppm}$, and $\mathrm{N}$-acetyl aspartate (NAA) peak at $2.0 \mathrm{ppm}$. MRI evaluation was done by two neuro-pediatric radiologists (R.I \& R.H.) who were blinded to the results of outcome assessment independently reviewed all MR images, and there was consensus between two reviewers for all the included cases. We did not include any case with disagreement in diagnosis. A radiological MRI scoring system was proposed to facilitate our interpretation of the imaging findings. A multiparametric MRI score (1-12) was calculated for 15 cases only who did the whole multi-parametric study depending on as follows: the location, (thalami, basal ganglia, WM of both cerebral and cerebellar hemispheres, brain stem), the severity of the disease, (hemorrhage, necrosis, and edema), diffusibility on DWI and ADC, and metabolites level on MRS (lipid/ lactate, gluatamine/ glutamate, and NAA peaks). One point was awarded for each of these features. Regarding the prognosis and outcome assessments were done by an experienced pediatric neurologist. The outcome of each patient was assessed according to 3 functions: physical function, social function, and his general health status. Four outcome categories were recognized and were assigned an alphabetical value: A, good, complete resolution, and $100 \%$; B, fair and mild to moderate degree of neurological sequelae, $\geq 50 \%$; $C$, worse and severe degree of neurological sequelae < $50 \%$; and D. poor and dead.

\section{Statistical analysis}

Data were coded and entered using the statistical package SPSS (Statistical Package for the Social Sciences) version 25. Data was summarized using mean, standard deviation, minimum and maximum in quantitative data, and using frequency (count) and relative frequency (percentage) for categorical data. The MR score and the clinical outcome category of the patients were both analyzed. Pearson correlation coefficient test was used for statistical analysis.

\section{Results}

A total of 30 children with ANEC, evaluated during autumn and winter seasons between October 2018 and March 2020, were retrospectively reviewed. The subjects 
consisted of 20 boys and 10 girls whose ages ranged from 8 months to 9 years (mean age, 4.32 years).

Clinical characteristics of the children enrolled in the study are summarized in (Table 1). All had prodromal symptoms appear in the first stage; the main symptom was fever presented in 26 cases (87\%), signs of upper respiratory tract infections presented in 10 cases (33.3\%), and gastroenteritis symptoms presented in 6 cases (20\%), and the least was the skin manifestation presented in 2 case (7\%) only. With the development of ANEC in the mid stage, rapid and progressive symptoms of brain dysfunctions presented as generalized convulsions and disturbance of consciousness in 18 cases $(60 \%)$; however, focal neurological deficits presented in 24 cases $(80 \%)$ and abnormal movements in 14 cases (46.6\%). At the follow-up stage of cases, the clinical outcome was moderate in the most of cases, 20 cases $(66.7 \%)$, full recovery (good) in 6 cases (20\%), and unfortunately 4 cases (13.3\%) died in ICU.

The multi-parametric MRI examination findings are illustrated in (Table 2). Concerning the location of lesions, the thalami were involved showing bilateral

Table 1 Clinical characteristics of the children diagnosed with

\begin{tabular}{lll} 
ANEC & & \\
\hline Stages & $\begin{array}{l}\text { NO. of } \\
\text { cases }\end{array}$ & $\%$ \\
\hline 1st stage (prodromal stage) & & \\
$\quad$ Respiratory manifestations (e.g., cough/ & 10 & $33.3 \%$ \\
expectoration) & & \\
GIT manifestations (e.g., vomiting, diarrhea) & 6 & $20 \%$ \\
Skin manifestations (e.g., erythema) & 2 & $7 \%$ \\
Fever associated with the previous & 14 & $47 \%$ \\
manifestations & & \\
$\quad$ Non-specific febrile illness & 12 & $40 \%$ \\
2nd stage (acute necotizing encephalopathy stage/neurological \\
manifestations)
\end{tabular}

Table 2 Conventional MRI findings regarding the extension of ANEC in children

\begin{tabular}{ll}
\hline The extension/location & No./\% \\
\hline Thalami & $30(100 \%)$ \\
Basal ganglia & $4(13.3 \%)$ \\
Brain stem & $24(80 \%)$ \\
WM of cerebral hemispheres & $22(73.3 \%)$ \\
$\quad$ Site & \\
$\quad$ Subcortical & $8(26.6 \%)$ \\
$\quad$ Deep periventricular WM & $14(46.7 \%)$ \\
Size & \\
$\quad$ Confluents & $6(20 \%)$ \\
$\quad$ Foci & $2(6.6 \%)$ \\
$\quad$ Sheets & $14(46.7 \%)$ \\
WM of cerebellar hemispheres & $10(33 \%)$ \\
Contrast-enhanced MRI & \\
Enhancement & $22(73 \%)$ \\
DWI/ ADC & \\
Diffusion restriction & $24(80 \%)$ \\
SWI & \\
Hemorrhage & $10(62.5 \%)$ \\
MRS & $14(87.5 \%)$ \\
- - N Glutamate/glutamine peak & \\
\hline
\end{tabular}

symmetrical thalamic involvement in all 30 case patients $(100 \%)$, brain stem in 24 cases $(80 \%)$, basal ganglia in 4 cases $(13.3 \%)$, cerebral WM in 22 cases $(73 \%)$, and cerebellar WM in 10 (33\%) (Fig. 1) by assessing SWI hemorrhage presented in 26 patients (86.7\%) (Fig. 2), cavitation in 4 cases (13.3\%) (Fig. 1), and edema in 24 cases (80\%) (Figs. 3 and 4). Diffusion restriction presented in 24 patients (80\%), enhancement presented in 22 cases (73\%) (Fig. 4), MRS shows high lipid/ lactate peak in all 15 examined cases (100\%) (Fig. 1), high gluatamine/ glutamate peak in 4/15 (26.7\%) (Fig. 1),iand reduced NAA peak in $8 / 15$ (53.3\%) (Fig. 3). Regarding the MRI scoring, 2 cases had score 9, 2 cases had score 8, 3 cases had score 7, 5 cases had score 6,2 case had score 3 , and 2 cases attained score 2 .

Upon correlating the prognosis with the MRI score, score 9 attained D outcome category (poor, dead), scores 8 and 7 attained $C$ outcome category, had worse sequelae, while score 6 attained B outcome category, and they had moderate sequelae, in the meanwhile, scores 3 and 2 attained A outcome category which represent the better prognosis, full recovery with no sequelae (Table 3). Statistically positive correlation $(r=0.1198)$ was found between the MR score and the outcome category. 

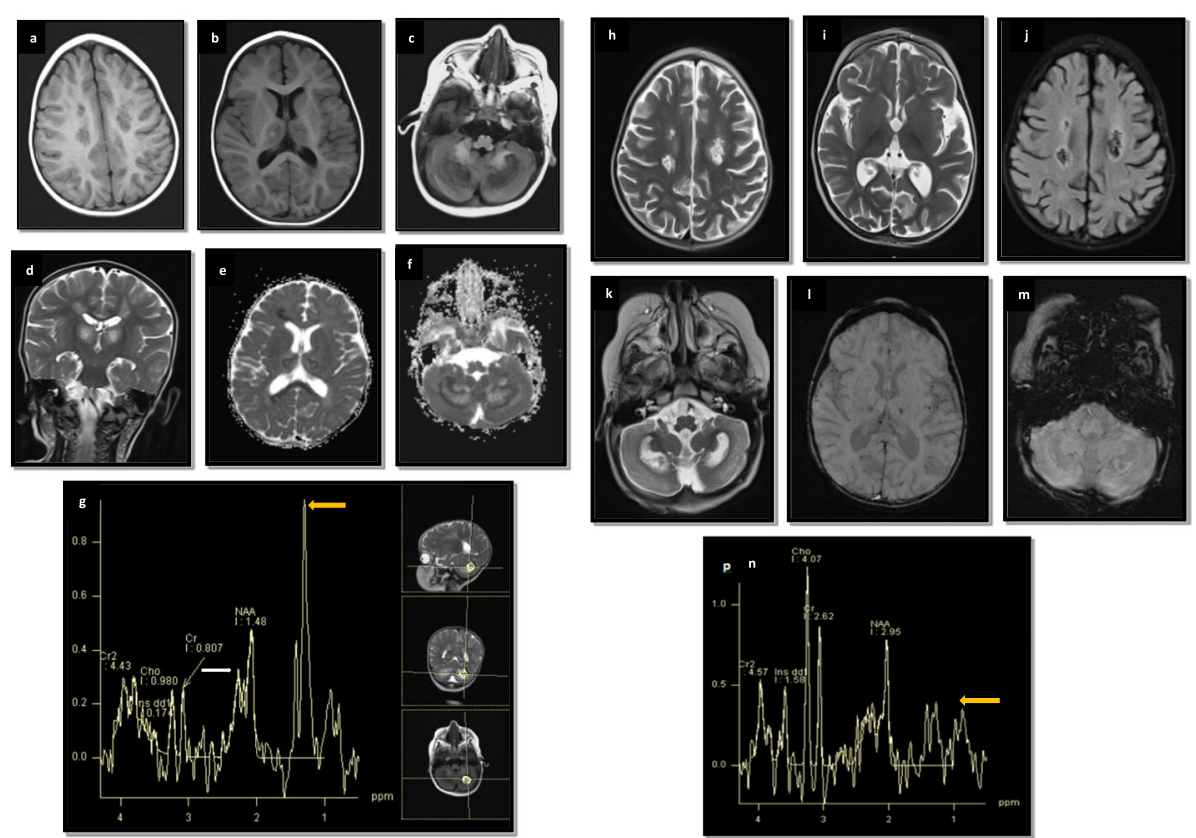

Fig. 1 Multi-parametric MRI dynamic changes of the brain of a 1-year-old patient who was left with marked sequelae. He presented with behavior changes, abnormal movement, and agitation and was diagnosed with ANEC. At the onset of the disease, a, $\mathbf{b}$, and $\mathbf{c}$ axial T1 WI, d coronal T2 WI show lesions on bilateral thalamic, basal ganglia (caudate nucleus), WM cerebral parenchyma (corona radiata), and WM cerebellar parenchyma being low signal in T1WI with small focus in the center of the thalamic lesions, high signal in T2WI. e and $\mathbf{f}$ Axial ADC shows typical tricolor pattern of thalamic lesions being high signal at the center, low surrounding signal, and bright peripheral signal; these also seen at cerebellar WM lesion. $\mathbf{g}$ Single-voxel MR spectrogram (TE, 115) of cerebellar WM lesion shows increased glutamate/glutamine complex peak intensities at $2.3 \mathrm{ppm}$ and lipid/lactate complex peak intensities at $1.2 \mathrm{ppm}$, with reduced NNA peak at $2 \mathrm{ppm}$. Follow-up after 4 weeks, $\mathbf{h}$ and $\mathbf{i}$ axial T2 WI, $\mathbf{j}$ and $\mathbf{k}$ FLAIR images, and reveal disappearance of WM cerebral lesions, generalized atrophy of the cerebral hemispheres, with widened ventricular size, impressive regression of the thalamic and cerebellum lesions being replaced by encephalomalacia surrounded by gliosis. I and $\mathbf{m}$ SWI shows hemosiderin deposition in the bilateral thalami and the cerebella (red arrow). $\mathbf{n}$ MRS shows dramatic reduction of the lactate peak (arrow) and the NAA peak returns within the normal range

\section{Discussion}

The distinctive neuro-radiologic manifestations of ANEC are multifocal, symmetric brain lesions with characteristic distributions which are remarkably similar among patients with ANEC, including bilateral thalami (typically present in all patients, serving as a distinctive feature), basal ganglia, brain stem, cerebral, and cerebellum WM [13]. Spinal cord may occasionally be involved as well [14]. During the clinical course of the disease, the neuro-radiologic manifestations are characterized by dynamic changes corresponding to their pathophysiological changes from edema to petechial hemorrhage and then to necrosis [1]. ANEC is a progressive and devastating disease, regardless of treatment. The prognosis varies from complete recovery to death. This article emphasizes on the clinical and radiological traits of ANEC. It proposes a multi-parametric MRI scoring and assess whether it correlates with patients' prognosis and outcome.

Here in this study, we demonstrate the dynamic change at the onset and during follow-up of the disease (mean 6-week intervals), in the clinical features and multi-parametric MRI brain imaging findings of the 30 children diagnosed with ANEC. A total of 30 children with ANEC, they consisted of 20 boys and 10 girls whose ages ranged from 8 months to 9 years (mean age, 4.32 years) with different clinical manifestation. All had prodromal symptoms appear in the first stage; the main symptom was fever presented in 26 cases $(87 \%)$ in the form of non-specific febrile illness presented in 12 cases (40\%) and fever associated with other manifestations presented in 14 cases (47\%), signs of upper respiratory tract infections in the form of flu symptoms, cough and expectoration presented in 10 cases $(33.3 \%)$ and gastroenteritis symptoms in the form of vomiting and diarrhea presented in 6 cases (20\%), and the least was the skin manifestation in the form of erythema in 2 cases (7\%) only. This was in accordance with study conducted by Seo et al. [6]; they reported that $100 \%$ of cases had precedent illnesses and 83\% had fever. With the development of ANEC in the mid stage, rapid and progressive symptoms of brain dysfunctions presented as generalized convulsions and disturbance of consciousness in 18 cases (60\%); however, focal neurological deficits 

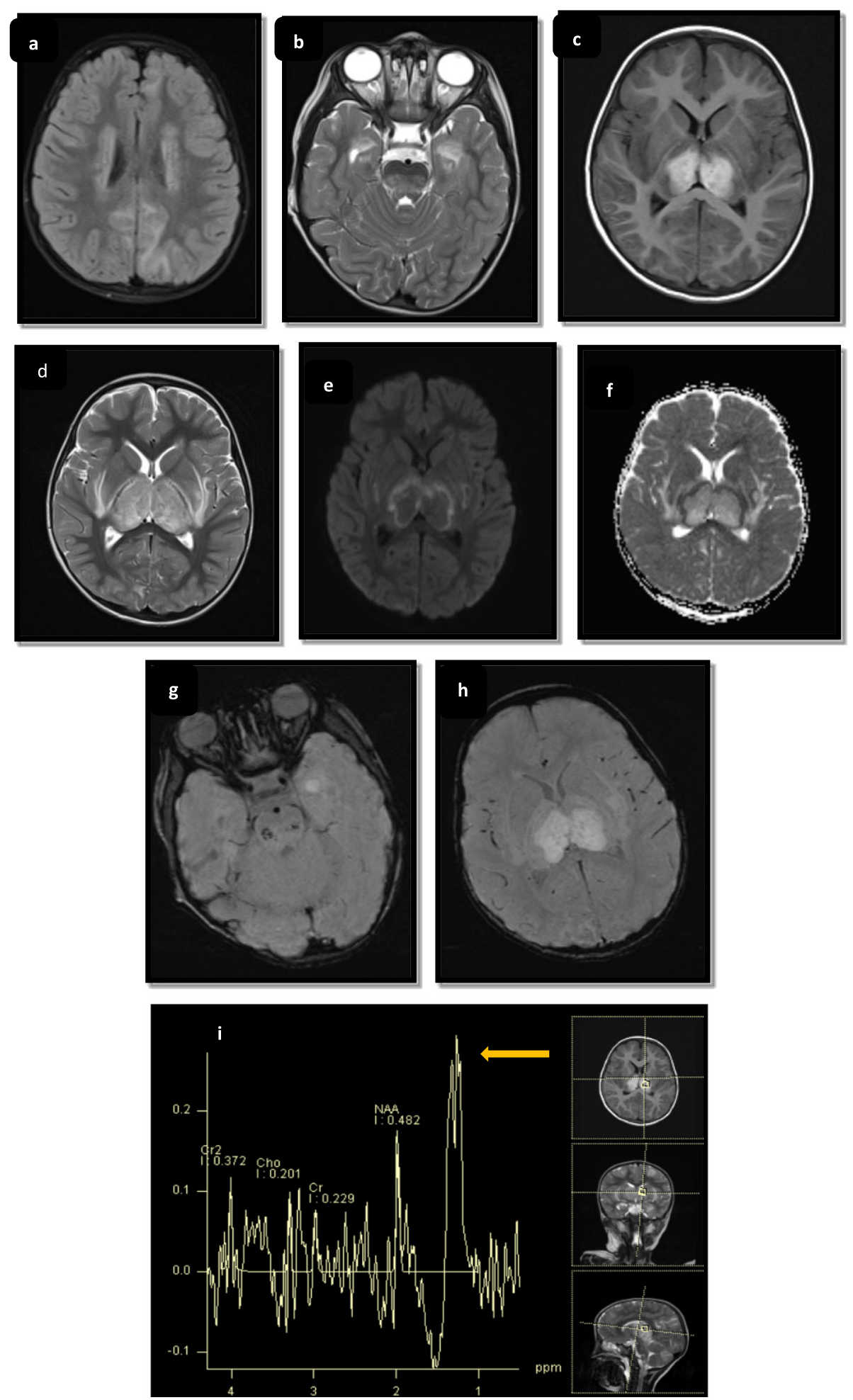

Fig. 2 (See legend on next page.) 
(See figure on previous page.)

Fig. 2 Multi-parametric MRI dynamic changes of the brain of a 3-year-old patient who died; on admission, he presented with respiratory disorders, fever, intractable convulsion, then DCL. He was diagnosed with ANEC. Axial images: a and b FLAIR, c T1 WI, d T2 WI, which reveal bilateral thalamic swelling with altered signal intensities being hyperintense in T2WI and FLAIR, and also bright signal in T1WI likely hemorrhage, a similar bilateral symmetrical areas of signal alteration are seen implicating the posterior limb of internal capsule, external capsule, putamia, periventricular cerebral WM, and posterior aspect of pons. e and $\mathbf{f} D W I$ and ADC show typical tricolor pattern of thalamic lesions showing peripheral restriction. $\mathbf{g}$ and $\mathbf{h}$ SWI shows foci of brain stem hemosiderin deposition; however, no signal loss on thalamic lesion. i Single-voxel spectroscopy with the region of interest being on the right thalamus and left lentiform nucleus with (TE, 115). Reduced NNA peak at 2 ppm, with a large lipid/lactate peak at $1.3 \mathrm{ppm}$ (arrow)

presented in 24 cases $(80 \%)$ in the form of behavioral changes presented in 8 cases $(26.7 \%)$, weak lower limbs presented in 6 cases (20\%) with hypotonia, hyporeflexia and inability to sit or stand, diplopia presented in 6 cases (20\%), and dysarthria presented in 4 cases (13.3\%). Seo et al. [6] found the neurological symptoms included mental change in $83 \%$, seizures, and focal neurological signs in 50\%. After clinical follow-up of cases to record the prognosis, the clinical outcome was moderate in most of the cases, 20 cases $(66.7 \%)$ being survived with neurological sequelae, (half of cases had moderate sequelae and the other half had sever sequelae), full recovery (good) in 6 cases (20\%), and unfortunately worse outcome in 4 cases (13.3\%) who died in ICU. A study conducted by Wong et al. [8] recorded that most of the patients about $66.7 \%$ fell into the moderate category, with fewer patients about $16.7 \%$ in the good and $16.7 \%$ in poor categories, and with no mortality.

By assessing the multi-parametric MRI examination, by conventional MRI using T1 WI, T2WI, and FLAIR sequences to assess the location of lesions, it revealed bilateral symmetrical thalamic involvement in all 30 cases patients $(100 \%)$, basal ganglia in 4 cases $(13.3 \%)$, brain stem in $24(80 \%)$, cerebellar WM in $10(33 \%)$, and cerebral WM in $22(73 \%)$ distributed regarding their site as follows: most of them at the deep periventricular WM in 14 cases $(46.7 \%)$ and 8 cases (26.6\%) affected subcortical WM and described regarding their size as sheets in 14 cases (46.7\%), confluents in 6 cases (20\%), and foci in just 2 cases (6.6\%). Tabarki et al. [15] stated that lesions in the brain are edematous and combined with mass effect at the onset of ANEC, so hypo-intensities are seen on T1 WI in most patients and these lesions were more clearly on FLAIR image. In the current study, all cases had brain lesions hypo-intensities by T1 WI, being hyper-intense in T2 WI and FLAIR imaging.

By considering functional MRI, just 24 cases $(80 \%)$ had edema and swelling with mass effect at the onset of the disease imaging with diffusion restriction being hyper-intense on DWI and hypo-intense on ADC, with restricted water diffusibility. The severity of the lesion was assessed by reporting; hemorrhage presented in 26 patients $(86.7 \%)$, cavitation in $4(13.3 \%)$, and edema in 24 cases (80\%). In the current study, by assessing SWI it demonstrated hemosiderin deposition not only in the thalami, but also in the brain stem and cerebellum; 13/ $15(86.7 \%)$ showing hemorrhage, even if not detected in conventional T1 WI. This is in agreement with a study conducted by Manara et al. [16] stated that small petechial hemorrhage is usually obscured by conventional MRI sequences; therefore, SWI is required which is more sensitive for the detection of the petechial hemorrhage.

Yadav et al. [17] reported that edema and mass effect would resolved gradually with petechial hemorrhage appeared, showed increased signal intensities (SI) in the center on T1 WI, while T2WI may reveal decreased SI surrounded by increased SI. In this study, follow-up MRI revealed partial to incomplete resolution, with regression of thalamic edema being diffusely smaller in size in comparison to imaging at the onset of disease, disappearance of hypo-intensity on the T1-WI and high SI on T2-W and FLAIR and DWI sequences, except for some cases revealed high signal in T2 WI being of low signal in T1WI and FLAIR representing necrosis with CSF like signal surrounded by gliosis.

A study done by Ormitti et al. [18] reported that the classical neuroimaging of ANEC was concentric, laminar, or tricolor appearance, it could be demonstrated in the thalami and the brain stem lesions being more obvious on ADC; the center of the lesion presents slightly high signal due to a perivascular hemorrhage and necrosis of neurons and glial cells occurs with a low signal in the surrounding of the lesion due to cytotoxic edema and acute swelling of oligodendrocytes, and its high signal in the periphery suggests extravasation and vasogenic edema. Regarding this study, this appearance observed in most of the patients; 22 cases (73.3\%), the rest of cases this appearance was not obvious.

Yoshida et al. [19] found that gadolinium-contrastenhanced MRI has been observed in identifying lesions at the very early stage of ANEC when conventional MRI, and even DWI and ADC show no abnormalities. This finding suggests that alteration of the blood brain barrier permeability might be the first step in the development of brain lesions. However, Wong and colleagues [8] found that not all ANEC patients presented contrast enhancement, partially due to the difference of performing 

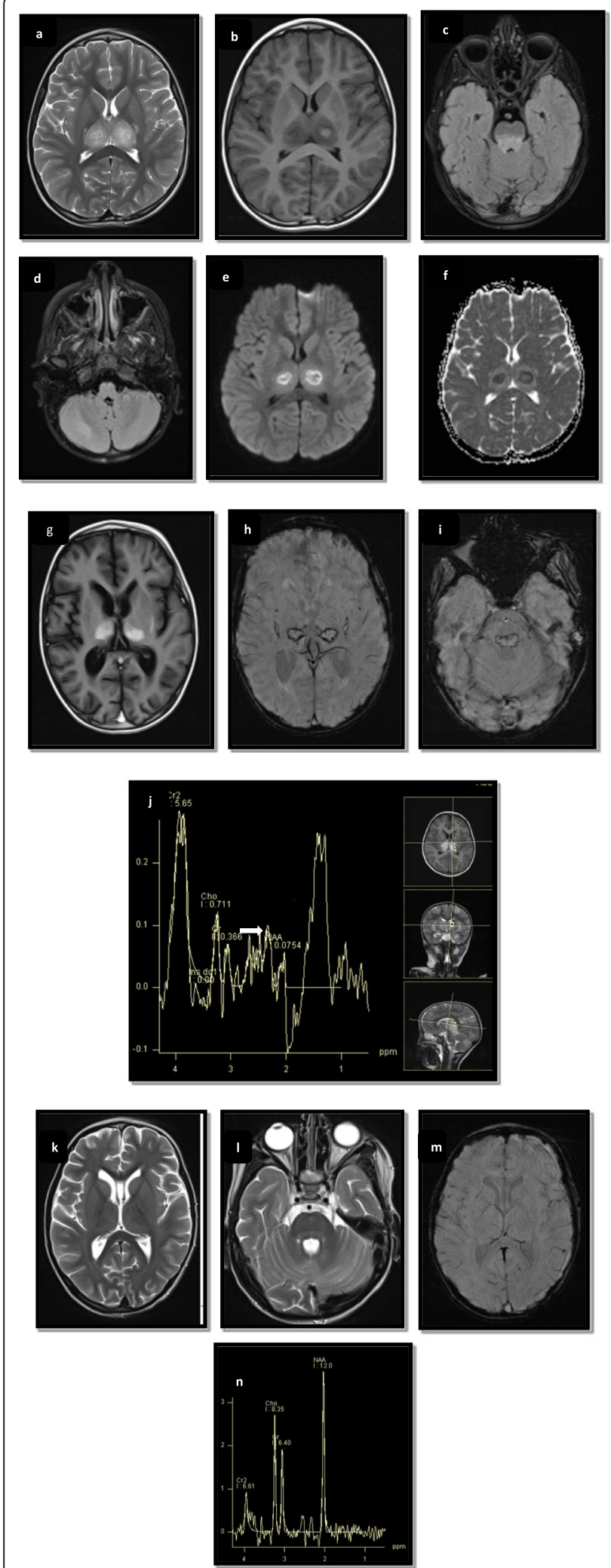

Fig. 3 Multi-parametric MRI dynamic changes of the brain of a 7year-old patient who recovered completely. He presented with fever, tonic colonic convulsions, then $\mathrm{DCL}$, behavior changes, abnormal movement, and agitation, was diagnosed with ANEC. At the onset of the disease, axial images (a), T1 WI (b), T2 WI (c and d) FLAIR show lesions on bilateral swollen thalamus, posterior limb of internal capsule, tegmen of pons, and right WM cerebellar parenchyma being low signal in T1WI with small focus of high signal in the center of the thalamic lesions (hemorrhage), heterogeneous high signal in T2WI, and FLAIR. e and $\mathbf{f} \mathrm{DWI}$ and ADC show peripheral restriction on DWI and typical tricolor pattern of thalamic lesions on ADC. g Contrast-enhanced T1WI shows bilateral thalamic homogenous enhancement. $\mathbf{h}$ and $\mathbf{i}$ SWI shows signal drop of hemosiderin deposition in the bilateral thalami and posterior brain stem. $\mathbf{j}$ Singlevoxel MRS (TE, 115) of left thalamic lesion shows reduced NNA peak, increased glutamate/glutamine complex peak intensities at $2.3 \mathrm{ppm}$ (arrow), and lipid/lactate complex peak intensities at $1.5 \mathrm{ppm}$. Broadening of the line-width may be caused by the occurrence of petechial hemorrhage within the lesion. Follow-up after 6 weeks, axial images ( $\mathbf{k}$ and $\mathbf{I})$ T2 WI reveal disappearance of WM cerebellar lesion, impressive regression of the thalamic size, and brain stem lesion with subtle bright signal. $\mathbf{m}$ SWI shows no signal drop. $\mathbf{n}$ MRS shows absence of the lactate and glutamate/glutamine peak and the NAA peak returns within the normal range

time and the severity of the disease; some unknown pathogenesis other than the alteration of BBB permeability may also exist. In the current study, this appearance observed in most of the patients; 22 cases $(73.3 \%)$, the rest of cases this appearance was not obvious.

There are few reports regarding DWI and MRS findings of ANEC in the literature. MRS has been applied to ANEC with a clinical significance; it is correlated either to the pathogenesis or to the prognosis. Aydin et al. [20] found a reversible or transient lipid-lactate and glutamate/glutamine complex peaks. The lipid-lactate complex peak might be due to the cell membrane damage or disintegration, while glutamate, a well-known excitatory neurotransmitter that may cause neuronal damage, might contribute to the pathogenesis by glutamatemediated exciteo-toxicity if excessive amounts were released into the synaptic cleft. A study conducted by Goo et al. [21] detected lipid-lactate complex peak and glutamate/glutamine complex peak on short TE MRS. Also, they found that the glutamate/glutamine complex peak was absent from some ANEC patients, indicating that the peak might depend on the severity of the disease and may serve as a predictor of outcomes. In this study, MRS was done for 15 cases, all cases (100\%) revealed transient high lipid-lactate peak suggestive of anaerobic glycolysis and cell membrane damage, reduced NAA peak in $8(53.3 \%)$ indicative of neuronal axonal damage; however, $4 / 15$ cases $(26.6 \%)$ had glutamate/glutamine complex peaks suggestive of glutamate-mediated exciteo-toxicity.

ANEC is a progressive and devastating disease, regardless of treatment. The prognosis varies from complete 

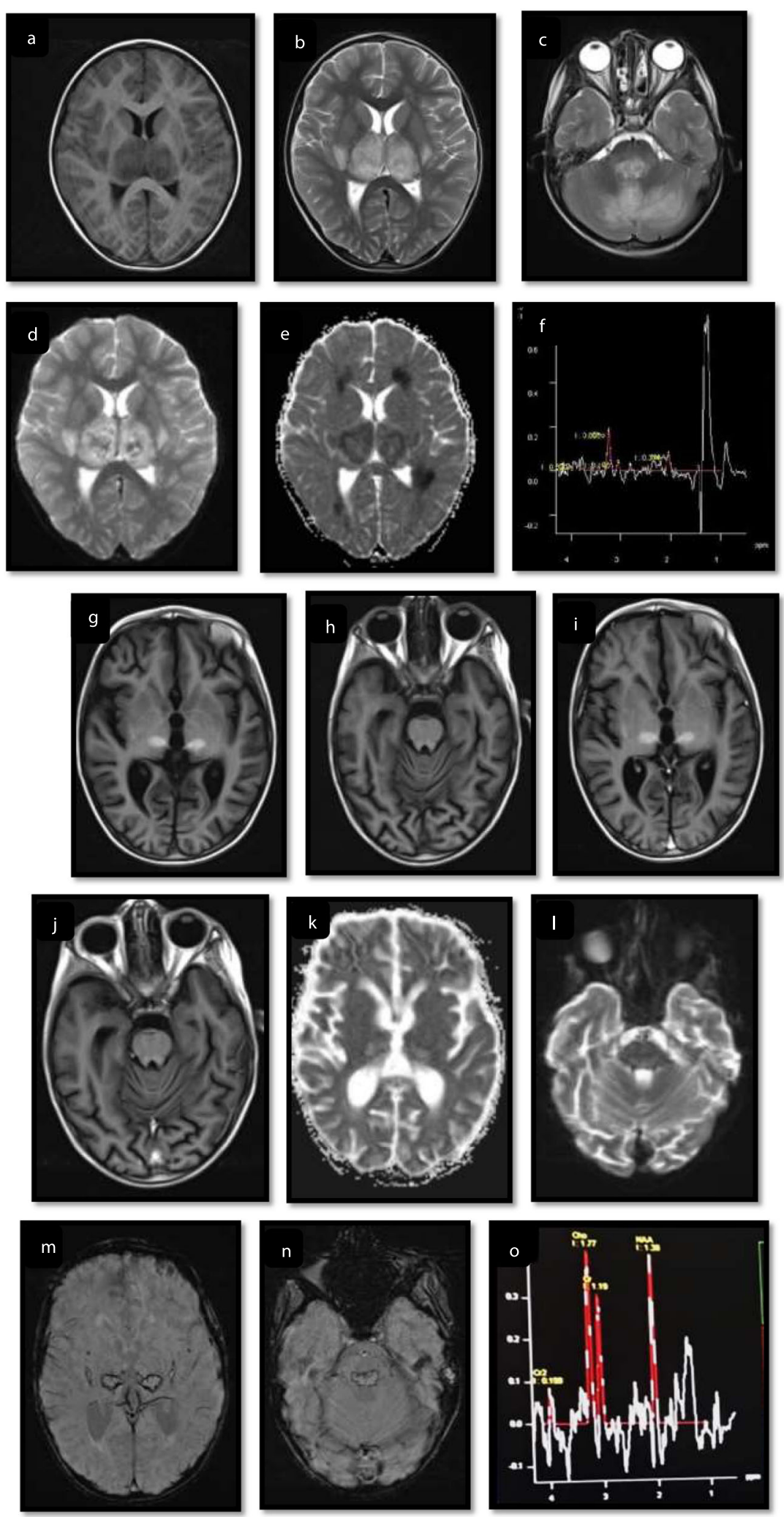

Fig. 4 (See legend on next page.) 
(See figure on previous page.)

Fig. 4 Multi-parametric MRI dynamic changes of the brain of a 5-year-old patient who was left with moderate sequelae. He presented with fever, tonic colonic convulsions, then DCL. He was diagnosed with ANEC. At the onset of the disease, axial images (a) T1 WI (b and c) T2 WI reveal lesions on bilateral swollen thalami, basal ganglia, and dorsal external capsule tegmen of pons, WM cerebral parenchyma and WM of both cerebellar parenchyma being low signal in T1WI, heterogeneous high signal in T2WI. $\mathbf{d}$ and $\mathbf{e}$ DWI and ADC show peripheral restriction on DWI and typical tricolor pattern of thalamic lesions on ADC. $\mathbf{f}$ Single-voxel MRS (TE, 115) of basal ganglia shows reduced NNA peak and increased lipid/lactate complex peak intensities at 0.8-1.5 ppm. Follow-up after 3 weeks, axial images $(\mathbf{g}$ and $\mathbf{h}) \mathrm{T1}$ WI reveal disappearance of WM cerebellar lesion, impressive regression of the thalamic size being of high signal (subacute hemorrhage), also regression of brain stem lesion, basal ganglia, and external capsule with subtle bright signal, moderate increased CSF spaces, and ventricular size (i and $\mathbf{j}$ ) contrast-enhanced MRI show no appreciable enhancement. $\mathbf{k}$ and I DWI shows restriction of pons lesions. $\mathbf{m}$ and $\mathbf{n}$ SWI shows signal drop in thalamic and brain stem lesions (hemosiderin). o Single-voxel MRS shows reduced the lactate peak and the NAA peak returns within the normal range

recovery to death. Lee and his brother [22] found that the mortality rate was about $30 \%$ and less than $10 \%$ of patients recovered completely, while more than $60 \%$ presented with neurological sequelae. Kim et al. [23] found that although patients had a good outcome however, the course of recovery was slow therefore, many children left hospital with significant neurologic problems. The reason for the good outcome may be due to early diagnosis of the mild form and prompt treatment of the disease [24]. Several factors are predictors for the prognosis of ANEC; the poor prognosis is with age below 1 year old and severely increased serum aminotransferase and protein in CSF [25]. Also, the presence of hemorrhage and localized tissue loss (e.g., localized atrophy or cystic encephalomalacia) would predict a poor prognosis [26]. Delirious behaviors, which result from brain stem lesions, were considered to be predictive of a poor outcome [27]. However, age above 4 years old and normal range or mild increased serum aminotransferase and protein in CSF [25]. The presence of asymmetric unilateral thalamic involvement and reversion of the image as well as absence of hemorrhage and localized tissue loss on MRI were regarded as a predictor of a good outcome [26]. Albayram et al. [28] concluded that patients who have restricted water diffusion in thalamic lesions on the ADC map, glutamate/glutamine complex, and increased lipid peak on short TE MRS generally have severe sequelae or high mortality.

Wong et al. [8] created a scoring system to evaluate patients with ANEC; they found that there was a significant correlation $(r=0.76)$ between the score and the outcome of the patients. Another ANEC severity scale by Yamamoto et al. [29] has been developed, including presence of shock, brain stem lesions, an age over 48 months, a platelet count below 100,000/휇 L, and an

Table 3 Neuroimaging finding regarding the severity of ANEC in children

\begin{tabular}{ll}
\hline The severity & No./\% \\
\hline Hemorrhage & $26(86.7 \%)$ \\
necrosis & $4(13.3 \%)$ \\
Edema $(\mathrm{T} 1 \mathrm{WI})$ & $24(80 \%)$ \\
\hline
\end{tabular}

elevated level of CSF protein; they suggested that this scale could be a predictor for the prognosis of children with ANEC. In the current study, CSF showed marked increased proteins in 8 cases; $4 / 8$ cases showed hemorrhagic necrosis in thalami and died in ICU. Of those who survived, 6/26 cases have recovered completely; however, the majority $20 / 26$ cases have permanent brain damage due to tissue necrosis, resulting in variable neurological sequelae from moderate to marked impairments in basic skills that may be regained over time, but the loss of brain tissue is permanent. Therefore, findings on MRI seem to allow us to predict the outcome.

In current study, MRI scoring encountered as follows: 2 cases had score 9, 2 cases had score 8,3 cases had score 7, 5 cases had score 6,2 cases had score 3 , and 2 cases attained score 2. Upon correlating the prognosis with the MRI score, score 9 (2 cases) attained D outcome category (poor, dead), scores 8 and 7 (2 and 3 cases respectively) attained $\mathrm{C}$ outcome category, had worse sequelae, while score 6 (5 cases) attained B outcome category, they had moderate sequelae, in the meanwhile, scores 3 and 2 ( 2 and 1 cases respectively) attained A outcome category which represent the better prognosis, full recovery with no sequelae. Upon correlating MRI score to the patient prognosis, we encountered the highest MRI scores were in the poor outcome category; whereas the patients with lower MR score (1 or 2) had better outcomes and statistically positive correlation $(r=0.1198)$ was found between the MR score and the outcome category; however, the relationship between the variables is week.

The study is assessing the diagnostic characteristics of the multi-parametric MRI of ANCE, including DWI, ADC, SWI, and MRS; it is the first study to assess all these techniques collectively in the same study. Also, the study is proposing a multi-parametric MRI scoring system correlating it with the outcome of children having ANCE (Table 4).

However, the study is limited by the fact that it included a limited number of cases because it is a rare disease. Also, MRI scoring and the clinical outcome show a weak positive correlation, this is may be due to limited 
Table 4 Correlation between MRI scoring and prognosis in children with ANEC (WM, white matter)

\begin{tabular}{lll}
\hline MRI scoring & No. of cases/\% & Prognosis \\
\hline 1 & 2 & $\mathrm{~A}$ \\
2 & 4 & $\mathrm{~A}$ \\
3 & 10 & $\mathrm{~B}$ \\
4 & 6 & $\mathrm{C}$ \\
5 & 8 & $2 \mathrm{C}, 2 \mathrm{D}$ \\
\hline
\end{tabular}

Good A, moderate B, worse C, and poor D (dead)

number of patients and in the other hand the devastating nature of the disease limiting the full multiparametric study to be done for all recruited cases because of bad clinical condition of the patients. Therefore, an extended study with sharing more number and early diagnosed cases would probably help in applying the multi-parametric MRI study to give a better idea regarding MRI score and prognosis correlation. Furthermore, the readers were from the same institution; therefore, some variability in results if other institutions replicated the work cannot be rolled out.

\section{Conclusion}

An extended multi-parametric MRI should be performed in ANEC, allowing early detection and scoring of the disease for better prognosis. There is a positive correlation between the clinical outcome and the multiparametric MR scoring.

\section{Abbreviations}

ANEC: Acute necrotizing encephalopathy of children; MRI: Magnetic resonance imaging; GIT: Gastrointestinal; CSF: Cerebrospinal fluid; WI: Weighted images; FLAIR: Fluid-attenuated inversion recovery; DWl: Diffusion-weighted images; ADC: Apparent diffusion coefficient; SWI: Susceptibility-weighted images; MRS: Magnetic resonance spectroscopy; ICU: Intensive care unit

\section{Acknowledgements}

The authors wish to acknowledge the assistance of pediatric neurology department staff, for their contribution to patient data collection.

\section{Authors' contributions}

"All authors have read and approved the manuscript." - Study concept: R.I., R.H., S.H.M., and E.E.S. Study design: R.H., R.I., E.W., and SH.M. Data acquisition: R.I., K.S.M., O.R., F.M., and Z.R. Data analysis and interpretation: R.H. and R.I. Statistical analysis: R.H. and R.I. Manuscript preparation: R.I., K.S.M., and O.R. Manuscript editing: R.I. Manuscript reviewing: R.I. and R.H.

\section{Funding}

Self-funding

\section{Availability of data and materials}

The datasets used and/or analyzed during the current study are available from the corresponding author on reasonable request.

\section{Ethics approval and consent to participate}

This study was approved by Radiology Department Kasr Alaini Cairo University hospital research ethics committee. Number: not available. Written informed consent was signed by patients' guardians.

\section{Consent for publication}

All patients' guardians included in this research gave written informed consent to publish the data contained within this study. Written informed consent for the publication of this data was given by their parent or legal guardian.

\section{Competing interests}

The authors declare that they have no competing interests.

\section{Author details}

${ }^{1}$ Radiology Department (Pediatric unit), Faculty of Medicine, Kasr El-Ainy Hospital, Cairo University, Kasr Al-aini Street, Al-manial District, Cairo, Egypt. ${ }^{2}$ Giza, Egypt.

Received: 31 March 2020 Accepted: 27 May 2020

Published online: 26 June 2020

\section{References}

1. Mizuguchi M, Abe J, Mikkaichi K, Noma S, Yoshida K, Yamanaka T et al (1995) Acute necrotizing encephalopathy of childhood: a new syndrome presenting with multifocal, symmetric brain lesions. J Neurol Neurosurg Psychiatry 58:555-561

2. Wang HS, Huang SC (2001) Acute necrotizing encephalopathy of childhood. Chang Gung Med J 24:1-10

3. Barkovich AJ (2005) Toxic and metabolic brain disorders. In: Pediatric Neuroimaging, 4th edn. Philadelphia, Lippincott Willams \& Wilkins, pp 76-189

4. Sugaya N (2000) Influenza-associated encephalopathy in Japan: pathogenesis and treatment. Pediatr Int 42:215-218

5. Kim YN, You SJA (2012) Case of acute necrotizing encephalopathy associated with para-influenza virus infection. Korean J Pediatr 55(4):147-150

6. Seo HE, Hwang SK, Choe BH, Cho MH, Park SP, Kwon S (2010) Clinical spectrum and prognostic factors of acute necrotizing encephalopathy in children. J Korean Med Sci 25(3):449-453

7. Yoshida T, Tamura T, Nagai Y, Ueda H, Shibata M, Awaya T (2013) MRI gadolinium enhancement precedes neuro-radiological findings in acute necrotizing encephalopathy. Brain Dev 35(10):921-924

8. Wong AM, Simon EM, Zimmerman RA, Wang HS, Toh CH, Ng SH (2006) Acute necrotizing encephalopathy of childhood: correlation of MR findings and clinical outcome. Am J Neuroradiol 27(9):1919-1923

9. Fasano A, Natoli GF, Cianfoni A, Ferraro D, Loria G, Bentivoglio AR et al (2008) Acute necrotizing encephalopathy: a relapsing case in a European adult. J Neurol Neurosurg Psychiatry 79(2):227-228

10. Ishida Y, Kawashima H, Morichi S, Yamanaka G, Okumura A, Nakagawa S et al (2015) Brain magnetic resonance imaging in acute phase of pandemic influenza A (H1N1) associated encephalopathy in children. Neuropediatrics 46:20-25

11. Wu X, Wu W, Pan W, Wu L, Liu K, Zhang H-L Acute necrotizing encephalopathy: an underrecognized clinicoradiologic disorder. Mediat Inflamm 792578

12. Williams TA, Brunsdon RK, Burton KLO, Drevensek S, Brady C, Dale RC, Mohammad SS (2019) Neuropsychological outcomes of childhood acute necrotizing encephalopathy. Brain Dev 41(10):894-900

13. Lyon JB, Remigio C, Milligan T, Deline C (2010) Acute necrotizing encephalopathy in a child with H1N1 influenza infection. Pediatr Radiol 40(2):200-205

14. Wolf K, Schmitt-Mechelke T, Kollias S, Curt A (2013) Acute necrotizing encephalopathy (ANE1): rare autosomal-dominant disorder presenting as acute transverse myelitis. J Neurol 260(6):1545-1553

15. Tabarki B, Thabet F, Al SS, Al Adwani N, Chehab M, Al Shahwan S (2013) Acute necrotizing encephalopathy associated with enterovirus infection. Brain Dev 35(5):454-457

16. Manara R, Franzoi M, Cogo P, Battistella PA (2006) Acute necrotizing encephalopathy: combined therapy and favorable outcome in a new case. Childs Nerv Syst 22(10):1231-1236

17. Yadav S, Das CJ, Kumar V, Lodha R (2010) Acute necrotizing encephalopathy. Indian J Pediatr 77(3):307-309

18. Ormitti F, Ventura E, Summa A, Picetti E, Crisi G (2010) Acute necrotizing encephalopathy in a child during the 2009 influenza A(H1N1) pandemia: MR imaging in diagnosis and follow-up. Am J Neuroradiol 31(3):396-400 
19. Yoshida T, Tamura T, Nagai Y, Hibata M, Kato T, Heike T et al (2013) MRI gadolinium enhancement precedes neuroradiological findings in acute necrotizing encephalopathy. Brain Dev 35(10):921-924

20. Aydin H, Ozgul E, Agildere AM (2010) Acute necrotizing encephalopathy secondary to diphtheria, tetanus toxoid and whole-cell pertussis vaccination: diffusion-weighted imaging and proton MR spectroscopy findings. Pediatr Radiol 40(7):1281-1284

21. Goo HW, Choi CG, Yoon CH, Ko TS (2003) Acute necrotizing encephalopathy: diffusion MR imaging and localized proton MR spectroscopic findings in two infants. Korean J Radiol 4(1):61-65

22. Lee JH, Lee M (2012) Recurrent acute necrotizing encephalopathy in a Korean child: the first non-caucasian case. J Child Neurol 27(10):1343-1347

23. Kim KJ, Park ES, Chang HJ, Suh M, Rha DW (2013) Novel influenza a (H1N1)associated acute necrotizing encephalopathy: a case report. Ann Rehabil Med 37(2):286-290

24. Vargas WS, Merchant S, Solomon G (2012) Favorable outcomes in acute necrotizing encephalopathy in a child treated with hypothermia. Pediatr Neurol 46(6):387-389

25. San MB, Teijeira S, Penin C, Garcia JL, Navarro C (2007) Acute necrotizing encephalopathy of childhood: report of a Spanish case. Pediatr Neurol 37(6): 438-441

26. Marco EJ, Anderson JE, Neilson DE, Strober JB (2010) Acute necrotizing encephalopathy in 3 brothers. Pediatrics 125(3):693-698

27. Okumura A, Mizuguchi M, Aiba H, Tanabe T, Tsuji T, Ohno A (2009) Delirious behavior in children with acute necrotizing encephalopathy. Brain Dev 31(8):594-599

28. Albayram S, Bilgi Z, Selcuk H, Selcuk D, Cam H, Koçer N et al (2004) Diffusion-weighted MR imaging findings of acute necrotizing encephalopathy. Am J Neuroradiol 25(5):792-797

29. Yamamoto H, Okumura A, Natsume J, Kojima S, Mizuguchi M (2014) A severity score for acute necrotizing encephalopathy. Brain Dev

\section{Publisher's Note}

Springer Nature remains neutral with regard to jurisdictional claims in published maps and institutional affiliations.

\section{Submit your manuscript to a SpringerOpen ${ }^{\circ}$ journal and benefit from:}

- Convenient online submission

- Rigorous peer review

- Open access: articles freely available online

- High visibility within the field

- Retaining the copyright to your article

Submit your next manuscript at $\boldsymbol{\nabla}$ springeropen.com 